branches to become familiar with the practical aspects of the matters with the statistics of which they are dealing.

The Memorandum also recommends transfer of personnel from departments to the Central Office and vice versa and, as already indicated, the staff of the latter should be available for assisting departments. The needs of certain departments for casual assistance should be met by providing the services of a mathematical statistician either by the appointment of a full-time officer, the loan of a suitable officer from the Central Statistical Office or the Department of Scientific and Industrial Research, the use of an advisory panel of experts, or by temporary engagement of a man from industry. The Memorandum particularly welcomes the interchange of expert personnel between industry and the Civil Service, and finally it is recommended that the Central Statistical Office or some other Government organization should ensure that the most effective use is made of modern machine methods.

The position of the Foreign and Colonial Offices, the Dominions Office and the India Office requires special consideration. In the Colonial Office at least, a statistical branch is recommended; but the responsibility for co-ordinating the trade statistics of the various Colonies should remain with the Board of Trade in view of the Board's experience in dealing with all trade statistics.

These proposals are parallel with those advanced on behalf of scientific workers generally, and which have commanded powerful suppurt in recent reports and in debates in the House of Lords. The Memorandum is also in line with recent suggestions for Civil Service reform, including the training and recruitment of staff. Pronouncements of members of the Government in regard to the importance of statistics and the remedying of admitted defects in the existing service warrant the hope that this notable technical contribution to the improvement of the machinery of government will receive the official recognition that it well deserves.

\section{A GAS-TUBE HARMONIC GENERATOR}

I $\mathrm{N}$ a search for methods of obtaining currents of high and closely controlled frequency for carrier systems, the Bell Laboratories recently investigated the harmonics generated by triggering gas-filled tubes. The studies showed that gas tubes can produce much higher frequencies than had been thought possible, if operated in a new circuit which accelerates the ionization and de-ionization of the tube. The harmonics thus generated provide standard frequencies so high as $\mathbf{2 5} \mathrm{mc}$. for calibrating oscillators. The sharp current pulses are useful in testing television systems, for making phase distortion measurements in co-axial cables, and in high-speed triggering devices.

A typical gas tube with grid control is a triode filled at low pressure with mercury vapour or argon gas. If the grid is maintained at a constant negative potential with reference to the hot cathode while the plate potential is increased with reference to the cathode, only a very minute electron current passes to the plate until a critical plate-cathode potential is reached. This critical potentialdepends on the structure of the tube, the gas used and the magnitude of the grid potential as well as on the past history of the tube. When the critical potential is reached, an arc forms and the tube current suddenly rises. The voltage across the tube simultaneously decreases if an external load is connected in series with the source, and the tube continues to conduct until the plate voltage falls below a minimum value necessary to maintain the arc. When the arc is extinguished, the gas ions in the tube migrate to the tube elements and the tube finally becomes de-ionized. As the tube becomes de-ionized, the grid again regains control and the above process may be repeated. The time required for de-ionization determines the frequency at which the foregoing cycle may be carried out.

In the new harmonic generator eircuit, the grid and plate are both supplied from the same A.c. source. This makes the plate voltage not only decrease to the value required to extinguish the tube, but also continue to a negative value. Likewise the grid voltage becomes negative. The negative potentials, primarily that on the grid, sweep the gas ions quickly from the tube, thus increasing the rate of de-ionization and the maximum frequency at which the tube can operate. An article by L. G. Kerota (Bell. Lab. Rec., 22, No. 2 ; October 1943) describes the new circuit, its operation and performance.

In one practical application the gas-tube harmonic generator is used to test the fidelity of televisionreceiving equipment. Because it generates sharp wave fronts of controllable duration, black-to-white signal changes of great rapidity can be obtained. For determining the phase shift of signals in co-axial cables, two gas-tube circuits operate synchronously. Signals from one are used locally, and those from the other are transmitted over a looped line. The phase shift is determined by observing the difference between the wave fronts of the two pulses.

\section{BIOLOGICAL RESULTS OF THE LAST CRUISE OF THE CARNEGIE*}

T HE present publication, the sixth of the series and the fourth devoted to biological reports, includes accounts of the phytoplankton by Herbert $W$. Graham, the marine algæ by William Albert Setchell, polychætous annelids by Aaron L. Treadwell, the mysids by W. M. Tattersall and, in addition, several smaller papers in various groups.

The phytoplankton samples, chiefly from the open ocean, were taken at the surface, $50 \mathrm{~m}$. and $100 \mathrm{~m}$., and collected by the Petterssen plankton pump. The main contents were diatoms and dinoflagellates. The area investigated is here divided for convenience into three regions: the southern region in the southeastern Pacific between South America and longitude $120^{\circ} \mathrm{W}$., the tropical region extending from Panama and Peru as far west as Guan, and the northern region between Yokohama and San Francisco. All were poor in phytoplankton. In general, the richest samples occurred in water of low temperature, lower salinity, lower $p \mathrm{H}$ and higher phosphate content, whereas little correlation could be found between numbers of plant cells and percentage saturation of oxygen, or between number of plant cells and quan. tity of dissolved silicate. An interesting condition

* Scientific Results of Cruise FII of the Carnegie during 1928-29 under Conmand of Captain J. P. Ault. Biology, IV. Department of Terrestrial Magnetism. (Pub. 555.) Pp. vi+92. (Washington, 